OPEN ACCESS

Edited by:

Chandra Ramakrishnan,

University of Zurich, Switzerland

Reviewed by:

Damer Blake,

Royal Veterinary College (RVC),

United Kingdom

Emanuel Heitlinger,

Leibniz Institute for Zoo and Wildlife

Research (LG), Germany

*Correspondence:

Françoise I. Bussière

Francoise.Bussiere@inrae.fr

${ }^{t}$ These authors have contributed equally to this work

Specialty section:

This article was submitted to

Parasite and Host,

a section of the journal

Frontiers in Cellular

and Infection Microbiology

Received: 23 November 2020 Accepted: 24 December 2020 Published: 04 February 2021

Citation:

Gaboriaud P, Sadrin G, Guitton E,

Fort $G$, Niepceron A, Lallier N, Rossignol C, Larcher T, Sausset A,

Guabiraba R, Silvestre A, Lacroix-Lamandé S, Schouler $C$, Laurent $F$ and Bussière FI (2021) The Absence of Gut Microbiota Alters the

Development of the Apicomplexan

Parasite Eimeria tenella.

Front. Cell. Infect. Microbiol. 10:632556.

doi: 10.3389/fcimb.2020.632556

\section{The Absence of Gut Microbiota Alters the Development of the Apicomplexan Parasite Eimeria tenella}

\author{
Pauline Gaboriaud ${ }^{1 \dagger}$, Guillaume Sadrin ${ }^{1 \dagger}$, Edouard Guitton ${ }^{2}$, Geneviève Fort ${ }^{1}$, \\ Alisson Niepceron ${ }^{1}$, Nathalie Lallier ${ }^{1}$, Christelle Rossignol ${ }^{1}$, Thibaut Larcher ${ }^{3}$, \\ Alix Sausset ${ }^{1}$, Rodrigo Guabiraba ${ }^{1}$, Anne Silvestre ${ }^{1}$, Sonia Lacroix-Lamandé ${ }^{1}$, \\ Catherine Schouler ${ }^{1}$, Fabrice Laurent ${ }^{1}$ and Françoise I. Bussière ${ }^{1 *}$ \\ 1 INRAE, Université de Tours, UMR ISP, Nouzilly, France, ${ }^{2}$ INRAE, UE PFIE, Nouzilly, France, ${ }^{3}$ INRAE, Oniris, PAnTher, \\ APEX, Nantes, France
}

Coccidiosis is a widespread intestinal disease of poultry caused by a parasite of the genus Eimeria. Eimeria tenella, is one of the most virulent species that specifically colonizes the caeca, an organ which harbors a rich and complex microbiota. Our objective was to study the impact of the intestinal microbiota on parasite infection and development using an original model of germ-free broilers. We observed that germ-free chickens presented significantly much lower load of oocysts in caecal contents than conventional chickens. This decrease in parasite load was measurable in caecal tissue by RT-qPCR at early time points. Histological analysis revealed the presence of much less first (day 2pi) and second generation schizonts (day 3.5pi) in germ-free chickens than conventional chickens. Indeed, at day 3.5pi, second generation schizonts were respectively immature only in germ-free chickens suggesting a lengthening of the asexual phase of the parasite in the absence of microbiota. Accordingly to the consequence of this lengthening, a delay in specific gamete gene expressions, and a reduction of gamete detection by histological analysis in caeca of germ-free chickens were observed. These differences in parasite load might result from an initial reduction of the excystation efficiency of the parasite in the gut of germ-free chickens. However, as bile salts involved in the excystation step led to an even higher excystation efficiency in germ-free compared to conventional chickens, this result could not explain the difference in parasite load. Interestingly, when we shunted the excystation step in vivo by infecting chickens with sporozoites using the cloacal route of inoculation, parasite invasion was similar in germ-free and in conventional chickens but still resulted in significantly lower parasite load in germ-free chickens at day 7pi. Overall, these data highlighted that the absence of intestinal microbiota alters E. tenella replication. Strategies to modulate the microbiota and/or its metabolites could therefore be an alternative approach to limit the negative impact of coccidiosis in poultry.

Keywords: Eimeria tenella, microbiota, germ-free, chicken, parasite, parasite invasion and development 


\section{INTRODUCTION}

Eimeria is an obligate intracellular parasite belonging to the phylum of Apicomplexa. In birds, various Eimeria species colonize the intestine with a localization that is specific to each species. Eimeria tenella (E. tenella) colonizes the caeca and is one of the most virulent species. It leads to haemorrhagic diarrhoea and high levels of morbidity and mortality in poultry flocks. Prevention and treatment of Eimeria infection are responsible for very high economic cost recently re-assessed to the level of about 13 billion dollars per year in the world (Blake et al., 2020). Current prophylaxis is based on anticoccidial drugs and vaccination, both with its advantages and limitations.

E. tenella invades and develops in intestinal epithelial cells. The in vivo parasite life cycle is divided in two parts: the endogenous asexual multiplication with three rounds of merogony and the sexual phase with the formation of macrogametes (female gametocytes) and microgametes (male gametocytes). Microgametes may fertilize a macrogamete resulting in the formation of a zygote (Ferguson et al., 2003). After maturation, it becomes an unsporulated oocyst released in the environment through the feces where it will develop into an infectious sporulated oocyst (exogenous phase or sporogony). Caeca are the richest part of the intestine in terms of bacteria abundance and diversity. Firmicutes, Bacteroidetes, and Proteobacteria are the main caecal bacterial phyla (Wei et al., 2013; Oakley et al., 2014; Qi et al., 2019). Importantly, E. tenella infection leads to alterations in the microbiota diversity and composition (Huang et al., 2018a). Taxa belonging to the order Enterobacteriaceae from the phylum Proteobacteria were increased in caecal contents from E. tenella infected chickens compared to those uninfected. Within the phylum Firmicutes, non-pathogenic bacteria Lactobacillus and Faecalibacterium were decreased while bacteria such as Clostridium perfringens are increased during the infection with $E$. tenella and are related to an increased occurrence of necrotic enteritis of chickens (Al-Sheikhly and Al-Saieg, 1980; Macdonald et al., 2017; Huang et al., 2018a; Huang et al., 2018b). The microbiota promotes the gut immune system maturation which in turn protect the host from enteric bacterial infections such as Salmonella infection (Crhanova et al., 2011). Studies have shown that the modulation of the chicken intestinal microbiota can limit Salmonella colonization (Stecher et al., 2010; Yang et al., 2014). In the case of an apicomplexan parasite such as Plasmodium falciparum, when Enterobacter is present in the gut, it confers a resistance to the parasite infection in Anopheles (Cirimotich et al., 2011a; Cirimotich et al., 2011b). However, other pathogens such as Histomonas meleagridis depend on bacteria for their growth in vitro (Ganas et al., 2012). Concerning E. tenella, most of the studies have focused on the effect of the microbiota on the clinical signs associated with infection (Visco and Burns, 1972a; Visco and Burns, 1972b; Bradley and Radhakrishnan, 1973; Lafont et al., 1975).

Abbreviations: GAM56, Macrogamete specific protein; FOA1, Flagellar Outer Arm protein 1
In the present study, we investigated the influence of the absence of microbiota on E. tenella infection. For this purpose, we used a recently developed model of germ-free fast-growing broilers (Guitton et al., 2020). Our results highlight a critical role for the microbiota in sustaining the optimal development of the parasite in the chicken intestinal tract. Strategies to modulate the composition of the microbiota and/or derived metabolites might therefore represent a promising strategy to improve the prophylaxis of coccidiosis.

\section{MATERIALS AND METHODS}

\section{Ethical Statement}

Animal experimental protocols were performed in accordance with the French legislation (Décret: 2001-464 29/05/01) and the EEC regulation (86/609/CEE) about laboratory animals. All chicken experiments were approved by the local ethics committee for animal experimentation of Centre Val de Loire (CEEA VdL nº19): 2018-04-26 (APAFIS N¹3904).

\section{Parasite}

The E. tenella INRAE PAPt36 strain (Et-INRAE) was used for all experiments except for studying the effect of the microbiota on parasite invasion. E. tenella strain was isolated from a poultry farming in France in 1974. Initially referenced as E. tenella strain PAPt38 is now renamed as E. tenella-INRAE strain. The strain was maintained by regular passages on chicken in the PFIE facility since then (Laurent et al., 2001). For parasite invasion studies, EtINRAE was transfected with nano luciferase and mcherry genes under the E. tenella actin promoter [Et-INV; (Yan et al., 2009; Swale et al., 2019)]. The mcherry gene allows sorting by flow cytometry of sporulated oocysts after inoculation of transfected sporozoites to chickens and for parasite amplification. The nano luciferase activity (NanoLuc ${ }^{\circledR}$ Luciferase, Promega) facilitates the detection of low load of parasites and accurate parasite quantification in tissues. Purification of sporozoites was performed as described by (Shirley, 1995). Briefly, $0.5 \mathrm{~mm}$ sterilized glass beads (Carl Roth, Karlsruhe, Germany) were added to sporulated oocysts. The oocyst wall was broken by vortexing for $17 \mathrm{~s}$. Released sporocysts were washed with PBS and incubated in standard excystation medium (trypsin $0.25 \%$ and biliary salts $0.5 \%$ in PBS; $\mathrm{pH} 7.4$ ) at $41^{\circ} \mathrm{C}$ for $1 \mathrm{~h}$. Sporozoites were then washed in PBS and ready for cloacal inoculation.

\section{Animals}

Conventional and germ-free Ross PM3 broilers were hatched in the Infectiology of Farm, Model and Wildlife Animals Facility (PFIE, Centre INRAE Val De Loire: https://doi.org/10.15454/1. 5572352821559333E12; member of the National Infrastructure EMERG'IN) as described by (Guitton et al., 2020). Briefly, Ross PM3 eggs from two French farms were collected, decontaminated with a $1.5 \%$ peracetic acid solution (1.5\% Divosan Plus VT53, Johnson Diversey, France). Eggs were then incubated, decontaminated a second time as described above and placed in a hatching incubator for production of conventional chicks or an 
isolator for the production of germ-free animals. Bacteriological controls were performed as described by (Guitton et al., 2020); animals were confirmed to be bacteria-free while conventional chickens developed a microbiota.

\section{Infection}

Two-week-old chickens were orally infected with different doses of sporulated oocysts of the Et-INRAE strain. At day 6 to 9 postinfection, chickens were euthanized by electronarcosis and caeca were collected. On caecal contents, oocysts were counted on MacMaster counting chambers. At day 2, 3.5, 5.5, and 7 post-infection, caecal tissues were washed and fixed in $4 \%$ formaldehyde (Laurypath, Chaponost, France) for histological analysis or directly frozen in liquid nitrogen and stored at $-80^{\circ} \mathrm{C}$ for gene expression analysis.

To study parasite invasion, the Et-INV strain was used. In order to shunt the natural excystation step in vivo, chickens were artificially infected by the cloacal route with $10^{6}$ sporozoites. After $16 \mathrm{~h}$ of infection, chickens were euthanized; caeca were removed and washed for measurement of the nano-luciferase activity (Promega). To investigate the parasite development in these conditions, chickens were cloacally infected with a lower dose of Et-INRAE sporozoites $\left(8 \times 10^{4}\right)$. At day 7 post-infection, chickens were euthanized and caeca were removed. Oocyst numbers in caecal contents were determined and caecal tissues were fixed or frozen as described above.

\section{Sporozoite Excystation Test}

For ex vivo sporozoite excystation test, the bile from the gall bladder and intestinal contents from different segments of the intestine of non-infected germ-free and conventional non-infected chickens were collected. Only intestinal contents were centrifuged at 7,000 $\mathrm{g}$ for $15 \mathrm{~min}$. Intestinal supernatants (dilution 1/10 in PBS) and bile (dilution $1 / 100$ in PBS) were incubated at $41^{\circ} \mathrm{C}$ for $30 \mathrm{~min}$ to $1 \mathrm{~h} 30$ with sporocysts obtained as described by (Shirley, 1995). As a control, the standard excystation medium described above was used. The number of sporocysts and excysted sporozoites were counted and the excystation efficiency was calculated as follows: (number of excysted sporozoites/2)/[(number of excysted sporozoites/2) + number of sporocysts)] x 100 .

\section{Histological Analysis}

Caecal tissues were collected, fixed in $4 \%$ formaldehyde (Laurypath) and embedded in paraffin wax (Leica). Tissue sections were stained with kit Masson Trichrome, light green variation (RAL diagnostics) or Hemalun Eosin Saffron.

\section{RT-qPCR}

Total RNA was isolated from caeca using TRIzol extraction (Life Technologies, Carlsbad, CA, USA). cDNA was synthetized using M-MLV Reverse Transcriptase (Promega, Madison, WI, USA), with random hexamer primers and oligo(dT)15 primer (Promega). cDNAs were then amplified by qPCR (CFX96, BioRad, Hercules, CA, USA) using $\mathrm{iQ}^{\mathrm{TM}} \mathrm{SYBR}^{\circledR}$ Green Supermix (Bio-Rad). Parasite load into caecal tissues was determined using specific primers to the E. tenella housekeeping genes Et18S, Etprofilin and Gallus gallus housekeeping genes g10 and gapdh (Table 1). Et18S is commonly used as a housekeeping gene during the parasite cycle (Walker et al., 2015); we added Etprofilin for which the expression was not modified in our experiment (data not shown). Microgamete and macrogamete gene expressions were determined using Etfoal, (microgamete-specific gene; ETH_00025255) and Etgam56, (macrogamete-specific gene, ETH_00007320) both obtained from ToxoDB release 34 (Table 1; Eurogentec, Seraing, Belgium) and the previously cited E. tenella housekeeping genes. The protocol used for qPCR was: $95^{\circ} \mathrm{C}$ for $5 \mathrm{~min}$ and $40 \mathrm{cycles}$ at $95^{\circ} \mathrm{C}$ for $10 \mathrm{~s}$ and $60^{\circ} \mathrm{C}$ for $15 \mathrm{~s}$ followed by $60^{\circ} \mathrm{C}$ for $5 \mathrm{~s}$. Melting curves were performed at $60^{\circ} \mathrm{C}$ for $5 \mathrm{~s}$ followed by gradual heating $\left(0.5^{\circ} \mathrm{C} / \mathrm{s}\right)$ to $95^{\circ} \mathrm{C}$. qPCR were performed in duplicate for each experiments. For parasite load, expression of Et18S and Etprofilin were normalized to Ct values obtained for Gallus gallus g10 and gapdh using the formula: $2^{-(\mathrm{Ct}}$ mean of Et housekeeping genes - Ct mean of gallus gallus housekeeping genes). For parasite microgamete and macrogamete gene expression, Etgam56 and Etfoal were normalized to Ct values obtained for Et $18 S$ and EtprofilinRNA using the formula: $2^{-(\mathrm{Ct} \text { Et gamete specific gene- Ct mean of }}$ Et housekeeping genes). Gene expression values are expressed as medians.

\section{Nano Luciferase Activity}

Caecal tissues were weighted, placed in lysis buffer (Tris $50 \mathrm{mM}$, EDTA $2 \mathrm{mM}$, Glycerol 10\%, Triton X-100 1\%) for $30 \mathrm{~min}$ at $4^{\circ} \mathrm{C}$ and then homogenized. After centrifugation (750 g, $5 \mathrm{~min}$ ), $25 \mu \mathrm{l}$ of supernatant were transferred to a 96-well plate and $25 \mu \mathrm{l}$ of buffer with substrate $(1 / 50)$ was added (Nano-Glo ${ }^{\circledR}$ Luciferase Assay System, Promega). Nano luciferase activity was measured using GloMax ${ }^{\circledR}$-Multi Detection plate reader (Promega).

\section{Statistical Analysis}

Data were analyzed using GraphPad Prism ${ }^{\circledR} 6(\mathrm{GraphPad}$ Software Inc., La Jolla, CA, USA). For data with only two

TABLE 1 | Sequences of primers used for RT-qPCR.

\begin{tabular}{|c|c|c|c|}
\hline Primer name & Accession number & Forward sequence $\left(5^{\prime}\right.$ to $\left.3^{\prime}\right)$ & Reverse sequence (5' to $\left.3^{\prime}\right)$ \\
\hline Et18S & 18S rRNA gene in Supercontig190 & CTGATGCATGCAACGAGTIT & GACCAGCCCCACAAAGTAAG \\
\hline Etprofilin & ETH_00010645 & GGAAGACGGAACCTCCATाT & CCAGAATCGCCACATCATAG \\
\hline Etfoa1 & ETH_00025255 & TCTCGCATTCCTCACAGATG & ATाTGCCTTGTGGATGAAC \\
\hline Etgam56 & ETH_00007320 & AGTGGCTGGAGAACTTCGTG & ATGCGGTTCGTGATCATGTC \\
\hline Gallus gallus g10 & 416492 & TCAAGGAAGGGTACGCTACA & AACAGCCTCTGCATCCACAGT \\
\hline Gallus gallus gapdh & 374193 & GTCCTCTCTGGCAAAGTCCAAG & CCACAACATACTCAGCACCTGC \\
\hline
\end{tabular}


groups of animals, a non-parametric Mann-Whitney test was performed. When more than two groups of chickens were compared, a non-parametric ANOVA followed by Dunn's multiple comparisons test was performed. When repeated measurements were performed, a non-parametric two-way ANOVA with multiple comparisons test was performed.

\section{RESULTS}

\section{Effect of the Absence of Microbiota on Oocyst Load in Caecal Contents and on Parasite Developmental Stages in Caecal Tissue}

To study the absence of microbiota on E. tenella infection, germ-free and conventional ROSS PM3 fast-growing broilers were infected with different doses of oocysts. The animals were euthanized and oocysts were counted in caecal contents at day 6,7 , and 9 days postinfection. We counted oocysts in caecal contents as it was technically not feasible to collect faeces daily for oocysts counts in our isolators and to maintain germ-free conditions concomitantly. Oocyst load were severely decreased in the caeca of germ-free chickens inoculated per os with a dose of 1,000 oocysts compared to conventional chickens at these different time points (day 6: 1,192fold decrease; day 7: 269-fold decrease; day 9: 129-fold decrease). Remarkably, decreasing the dose of inoculum to 100 oocysts for conventional animals was not enough to decrease their parasite load in caecal contents to the level of germ-free animals 6 days postinfection when they were inoculated per os 1,000 or 10,000 or even 50,000 sporulated oocysts (Figure 1A). However, inoculation of 10,000 sporulated oocysts led to a similar parasite load in caecal contents at day 7 post-infection for both germ-free and conventional chickens (Figure 1A).
Interestingly, lower parasite load in caecal tissues of germ-free chickens was already observed as soon as day 2 post-infection (Figure 1B). Indeed, histological analysis revealed less firstgeneration schizonts at day 2 post-infection and less secondgeneration schizonts at day 3.5 post-infection in germ-free compared to conventional chickens. At this later time point, second-generation schizonts were clearly smaller, immature and second generation merozoites were not formed yet in germ-free chickens compared to conventional chickens in which the secondgeneration schizonts were mature with clearly developed merozoites (Figure 2B). This observation suggests a delay in the beginning of the parasite replication phase in the absence of microbiota (Figure 2B). We next studied the expression of gamete specific genes Etfoal (for microgamete) and Etgam56 (for macrogamete) (Walker et al., 2015). For this purpose, gamete gene expressions were normalized to the parasite housekeeping genes (Etprofilin and Et18S). We observed reduced Etfoal and Etgam56 gene expressions in germ-free compared to conventional chickens at day 5.5 post-infection (Figure 2A). This result was corroborated by histological analysis showing a higher number of parasites in the stage of gametes in conventional compared to germ-free chickens at this time point (Figure 2B). At day 7 post-infection, the expression of both Etfoal and Etgam56 were similar for both groups (Figure 2A). Histological analysis revealed that the number of gametes decreased in conventional chickens at day 7 post-infection (Figure 2B) and increased in germ-free chickens. These data suggest a delay in E. tenella development as a result of a longer asexual phase in germ-free compared to conventional chickens.

\section{Bile From Germ-Free Chickens Increases Sporozoite Excystation}

When sporulated oocysts are ingested by chickens, mechanic and enzymatic activities such as trypsin and biliary salts are
A

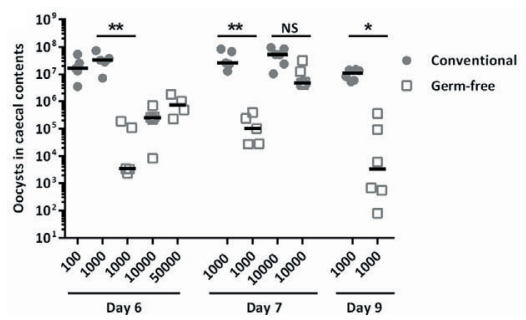

B

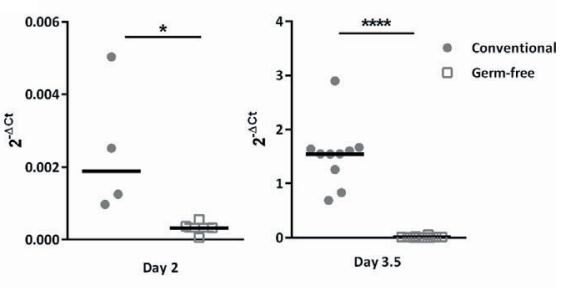

FIGURE 1 | The absence of microbiota reduced parasite load in caecal contents and in caecal tissues during infection. Conventional and germ-free chickens were orally infected with different doses of $E$. tenella. (A) Oocyst load in caecal contents. Oocyst load was evaluated at day 6 , 7 , and 9 post-infection. Medians are represented (One-Way ANOVA, Dunn's multiple comparisons test; $\mathrm{n} \geq 4$; ${ }^{*} P<0.05$; ${ }^{\star *} P<0.01$ between germ-free and conventional chickens). (B) The parasite load in caecal tissues is lower as soon as day 2 post-infection in germ-free chickens compared to conventional chickens. To be able to detect the parasite, conventional and germ-free chickens were orally infected with high doses of $E$. tenella (500,000 sporulated oocysts) for 2 days and 3.5 days of infection. The parasite load in caecal tissues was evaluated by RT-qPCR using the mean transcript expression of Et18S and Etprofilin relative to host housekeeping genes (Gallus gallus gapdh and $g 10$ ) using the $2^{-\Delta \mathrm{Ct}}$ formula: Ct Et housekeeping genes $-\mathrm{Ct}$ host housekeeping genes. Medians are represented (Mann-Whitney; $\mathrm{n} \geq 4$; ${ }^{\star} P<0.05$;

${ }^{* * *} P<0.0001$ between germ-free and conventional chickens). 
A

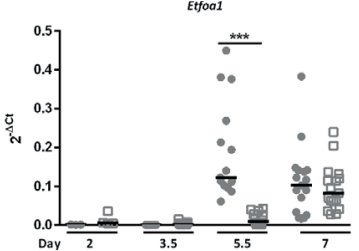

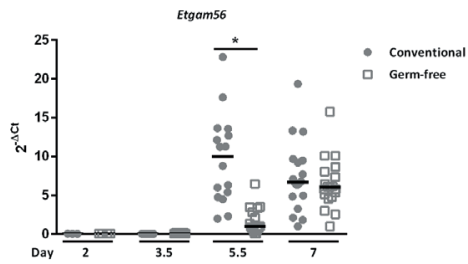

B
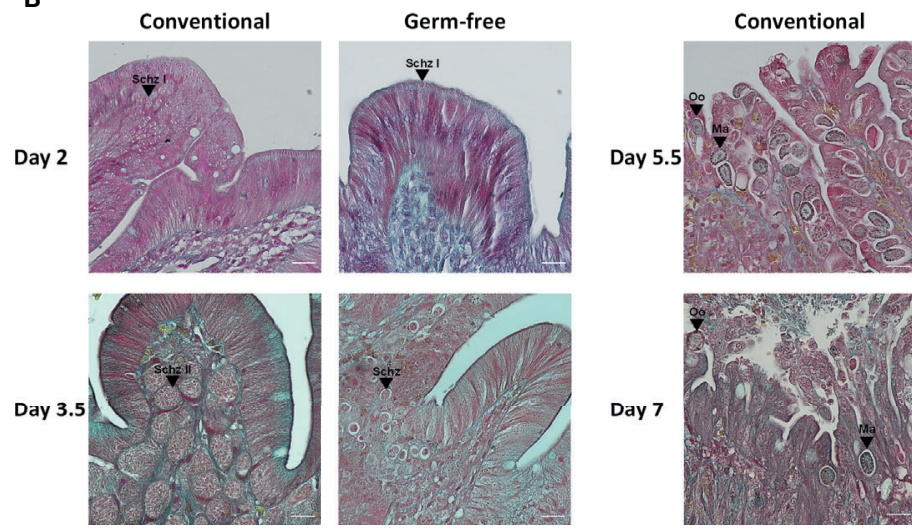

(2)

FIGURE 2 | The presence of immature second generation schizonts in germ-free chickens at day 3.5 post-infection suggests a delay in the development of the parasite. (A) Gametocyte gene expressions are delayed in germ-free chickens. Sporulated oocysts were orally inoculated to germ-free and conventional chickens (500,000 for 2 days and 10,000 for 3.5, 5.5, and 7 days of infection). Gametocyte gene expressions were detected by RT-qPCR on caecal tissues. The expression of microgamete and macrogamete specific genes (Etfoa1 and Etgam56) were determined by RT-qPCR and expressed relative to E. tenella housekeeping genes (Et18S and Etprofilin) at day 2, 3.5, 5.5, and 7 post-infection using the $2^{-\Delta C t}$ formula: Ct Et gametocyte specific gene - Ct Et housekeeping genes. Medians are represented (One-Way ANOVA, Dunn's multiple comparisons test; $\mathrm{n} \geq 3$; ${ }^{*} P<0.05$; ${ }^{* *} P<0.001$ between germ-free and conventional chickens). (B) Representative histological images showing a delay in parasite development in germ-free chickens. Histological analysis was performed on tissue sections stained with MassonGoldner Trichrome. Scale = $20 \mu \mathrm{m}$; Schz, schizont; Ma, macrogamete; Oo, oocyst.

necessary for sporozoite excystation. Sporozoites are then able to invade intestinal epithelial cells in which the parasite develops. Since lower parasite development in germ-free chickens may have resulted from a decrease in the excystation efficiency, we investigated the ability of sporozoites to excyst in the presence of intestinal contents and bile extracts from conventional and germ-free chickens. We then tested the activities of intestinal contents from specific segments of the gut and bile obtained from the gall bladder from germ-free and conventional chickens. We observed a higher excystation efficiency with duodenal and ileal supernatants combined with bile from germ-free chickens (Figure 3). To clarify the respective contribution of the intestinal content and the bile in this higher excystation efficiency, we used duodenal contents from germ-free or conventional chickens supplemented with bile from conventional, germ-free, or the standard excystation medium as a control. In these conditions, we observed a higher excystation efficiency when using duodenal contents from germ-free or conventional chickens combined with bile from germ-free chickens (Figures 4A, B). This result demonstrates that the higher excystation efficiency was likely to be related to the bile composition of germ-free chickens. However, this data cannot explain the lower parasite load observed caecal contents from germ-free compared to conventional chickens as a higher efficiency of excystation in germ-free chickens would be expected to lead to a higher infection rate.

\section{The Absence of Gut Microbiota Alters the Development of the Parasite but Not Its Capacity to Invade the Caecal Mucosa}

In order to study the effect of the absence of microbiota on sporozoite invasion in vivo, without the influence of the bile on the excystation efficiency, we shunted this process by cloacally infecting chickens with sporozoites excysted in vitro. Using $E$. tenella sporozoites expressing the nano luciferase reporter gene, we were able to determine parasite invasion level and showed that there was no difference in parasite invasion between conventional and germ-free chickens (Figure 5A). However, in the same experimental conditions when the animals were kept until day 7 post-infection, a decrease in oocyst load in caecal contents (280-fold) and in tissue parasite load revealed by RTqPCR and histological analysis were observed in germ-free compared to conventional chickens (Figures 5B, C). These results suggest a major role of the microbiota on the development of the parasite after the invasion process but not on the parasite invasion itself. 

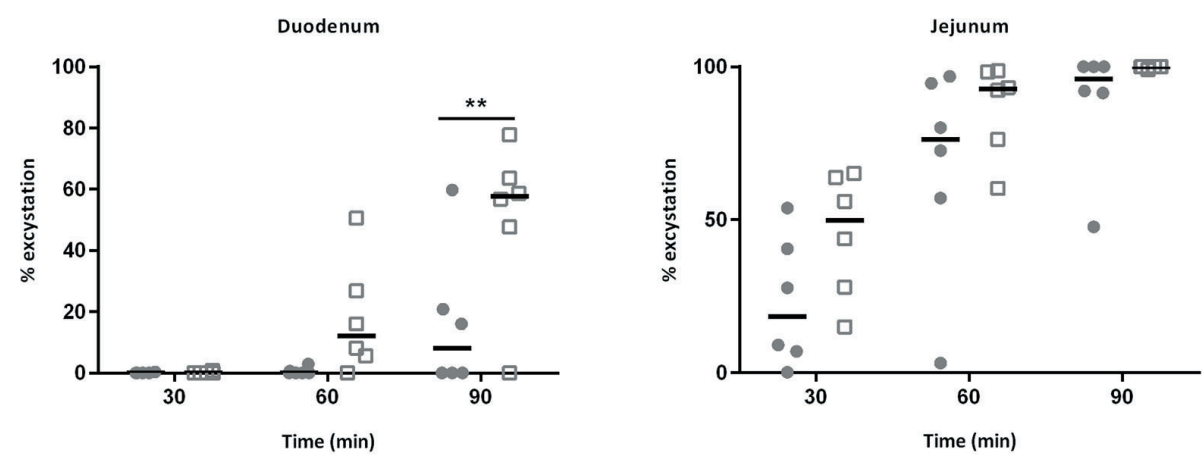

- Conventional $\square$ Germ-free
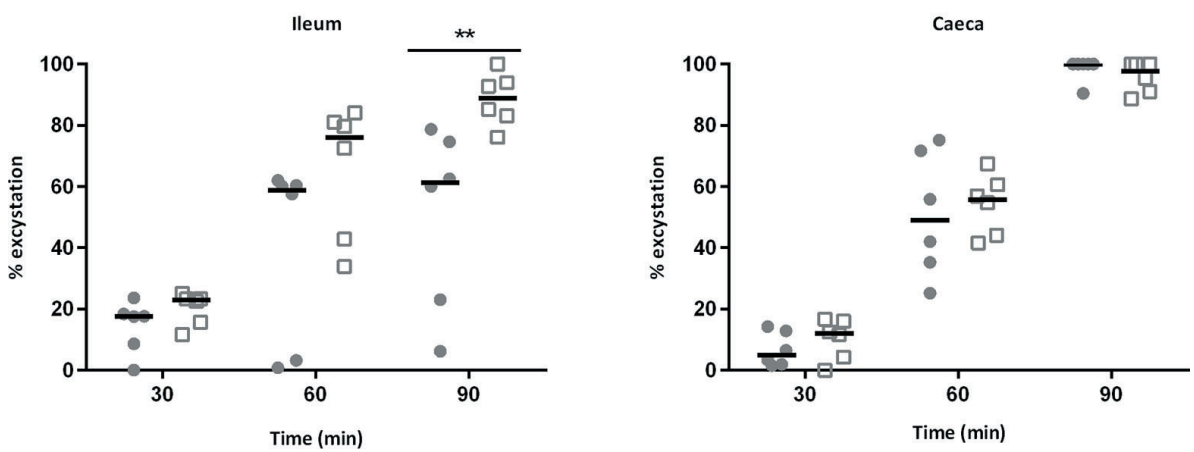

FIGURE 3 | The sporozoite excystation efficiency is increased with bile and duodenum or ileum contents from germ-free chickens. Sporozoites were excysted using contents of different segments of the intestine and bile from the same animals (germ-free and conventional chickens). The excystation efficiency of sporozoites is higher when incubating sporocysts with duodenal, ileal contents and bile from germ-free chickens. Medians are represented (Two-Way ANOVA, Sidak multiple comparisons test; $\mathrm{n}=6$; ${ }^{\star *} P<0.01$ between germ-free and conventional chickens).

\section{DISCUSSION}

To study the effect of the microbiota on E. tenella infection, we used an original model of germ-free broilers that we recently developed in our facilities (Guitton et al., 2020). A previous study reported that a high dose of E. tenella inoculum administered per os (>10,000 sporulated oocysts) led to similar oocyst load in caecal contents in germ-free and conventional chickens (Visco and Burns, 1972a). This observation may have resulted from a crowding effect resulting in maximal oocyst production. Indeed, we confirmed these findings, but we observed that with a lower inoculation dose of 1,000 sporulated oocysts administered per $o s$, germ-free chickens presented a significantly lower load in oocysts in caecal contents at three different time points over a 3 days period. A lengthening of the parasite life cycle was observed and characterized by a delay in the formation of first and second generation schizonts. In germ-free chickens, at day 3.5 post-infection, second generation schizonts were small and immature with merozoites that were not formed yet while in conventional chickens, second generation schizonts were large and mature with clearly developed merozoites as revealed by histological analysis. Consequently, this delay in the asexual phase development led to a delay in the formation of gametocytes. This delay observed by histological analysis was confirmed by the expression of gametocyte specific genes, Etgam56 for macrogametes and Etfoal for a flagellar outer arm protein 1 specific to microgametes at day 5.5 post-infection (Walker et al., 2015). This result suggests that a delay in the appearance of the gamogony stage associated to a reduced number of sexual stages most probably will result in a delayed and decreased oocyst load in germ-free chickens.

As we observed lower parasite load in caecal tissues at day 2 post-infection in germ-free chickens, we hypothesized that there could be an effect of the microbiota on the excystation step and/ or in the invasion process. As excystation media used in laboratory to artificially excyst sporozoites from sporocyst contains biliary acids, we used bile from each animal. When assessing the ability of sporozoites to excyst from sporocysts, an increased excystation efficiency was observed when using bile from germ-free chickens suggesting a difference in its 


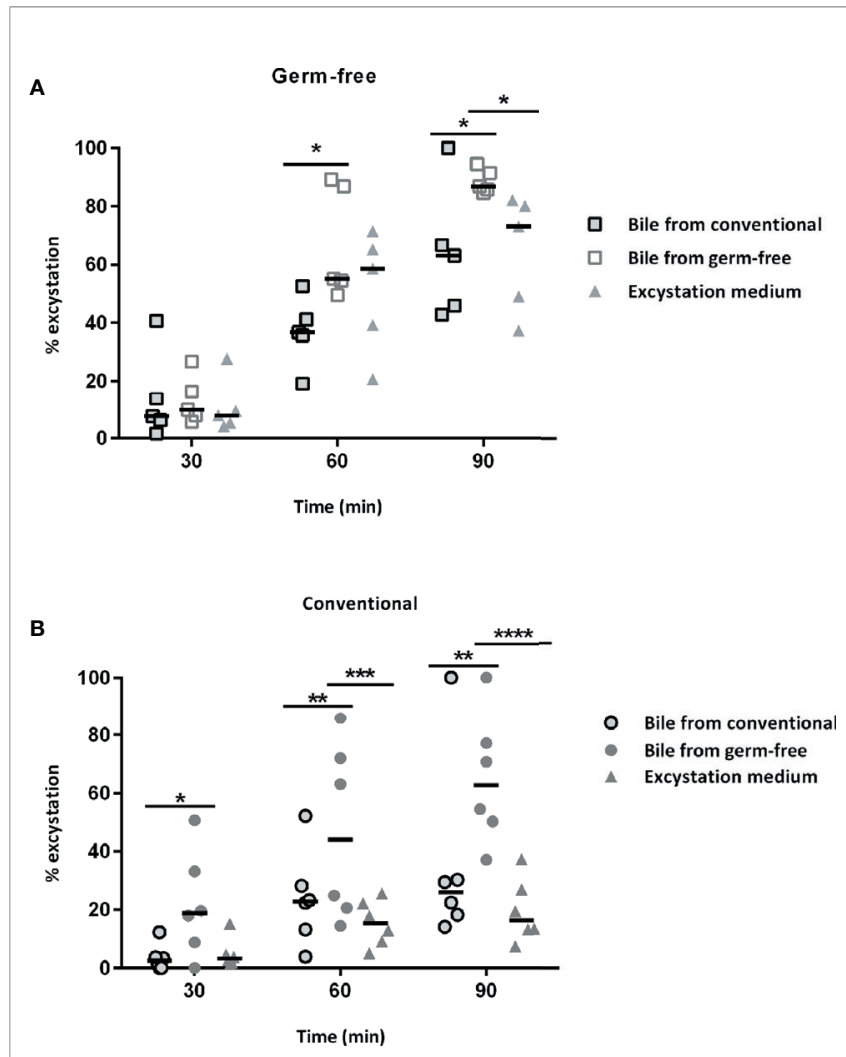

FIGURE 4 | The bile from germ-free chickens is responsible for the increase in the sporozoite excystation efficiency. (A) Bile from germ-free chicken leads to a higher excystation efficiency of sporozoites when incubating sporocysts with duodenal contents from germ-free chickens. Sporozoites were excysted when incubating sporocysts with duodenal contents from germ-free chickens and bile from conventional, germ-free chickens or excystation medium. Medians are represented (Two-Way ANOVA, Tukey multiple comparisons test; $n=5$; ${ }^{*} P<0.05$ ).

(B) Bile from germ-free chicken leads to a higher excystation efficiency of sporozoites when incubating sporocysts with duodenal contents from conventional chickens. Sporozoites were excysted when incubating sporocysts with duodenal contents from conventional chickens and bile from conventional, germ-free chickens or the standard excystation medium. Medians are represented (Two-Way ANOVA, Tukey multiple comparisons test; $\mathrm{n}=6$; ${ }^{\star} \mathrm{P}<0.05 ;{ }^{* *} P<0.01$; ${ }^{* *} \mathrm{P}<0.001$; $\left.{ }^{\star * * *} P<0.0001\right)$.

composition. In animals, biliary acids are synthetized from cholesterol in the liver. Conjugated bile acids (primary bile acids) are secreted in the intestine and are involved in lipid absorption. The presence of bacteria in the luminal content leads to deconjugation and dehydroxylation reactions and then to the formation of unconjugated bile acids and secondary bile acids (Long et al., 2017). Consequently, in the absence of a microbiota, the composition of the bile is different with mainly conjugated bile acids found in caeca of germ-free animals. (Madsen et al., 1976). This change in the bile composition and in particular of the biliary acids may explain the higher efficiency of excystation of sporozoites in the presence of bile from germ-free chickens. However, higher excystation efficiency in the absence of microbiota cannot explain the lower parasite load in caecal contents and in tissues found in germ-free compared to conventional chickens.

We then sought to investigate the ability of sporozoites to invade the caecal mucosa of germ-free or conventional chickens by using cloacal inoculation with sporozoites in order to shunt the excystation step. The microbiota helps in the appropriate development and maturation of the gutassociated lymphoid organs in chickens (Hegde et al., 1982). However, we observed lower parasite load in caecal contents and in tissues in germ-free chickens suggesting that the lack of maturation of the immune system in the absence of microbiota is probably not involved. Indeed, the microbiota also leads to a mature intestinal mucosa including goblet cells and their mucin production (Rolls et al., 1978) (Cheled-Shoval et al., 2014). Despite the fact that the caecal mucosa of germfree chickens may have different characteristics, we showed that the absence of microbiota did not modify the ability of sporozoites to invade the epithelium. However, when animals were cloacally infected and kept for 7 days, the oocyst load in caecal contents and the tissue parasite load were still lower in germ-free than in conventional chickens. Even though the dose of inoculum administered orally and cloacally cannot be easily compared, cloacal infection led to a decrease in oocyst load in caecal contents similar to the one observed with oral infection. This result demonstrates that the absence of microbiota alters the development of E. tenella but not the capacity of sporozoites to invade caecal epithelial cells.

The microbiota harbours a wide variety of bacteria which metabolize the nutrients of the digestive content and synthetizes metabolites that can act on the immune cells (Liu et al., 2019) and/or can be critical for parasite growth. As Eimeria infection disrupts carbon and nitrogen metabolism (Huang et al., 2018b), the parasite is likely to require some metabolites issued at least from these two biochemical pathways. We hypothesize that some metabolites synthetized by the microbiota might be critical substrates for parasite replication and are present in reduced amount in germ-free chickens to allow its normal growth. Alternatively, these metabolites can affect parasite host cell metabolism, the lining of intestinal epithelial cells, the mucosae and indirectly the development of the parasite. Further studies will be performed to compare the precise composition of metabolites in caecal contents from germfree and conventional chickens and to identify molecules that are important for the development of the parasite.

In conclusion, we revealed that the absence of microbiota alters the development of E. tenella. Strategies to modulate the composition of the microbiota and its metabolism would be of interest to inhibit parasite replication and/or to stimulate the immune response. As nutrition factors can play a major role in intestinal health and more particularly on its microbiota, it could have a beneficial effect on the outcome of the disease. Notably, innovative nutritional approaches or probiotics are societal acceptable approaches in face of growing parasite resistance to anticoccidials and lack of cost-effective prophylaxis. 
A

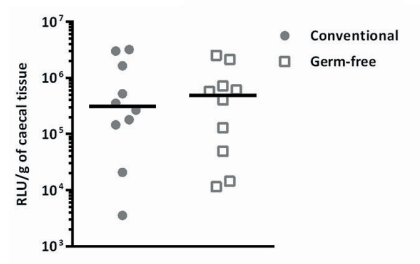

B

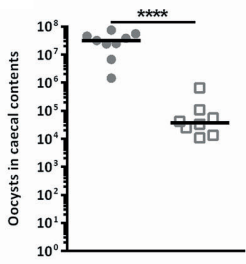

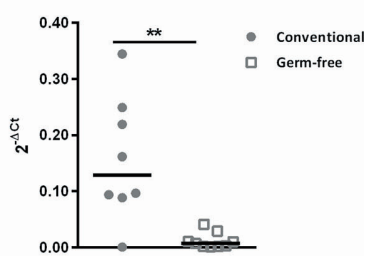

C

Conventional
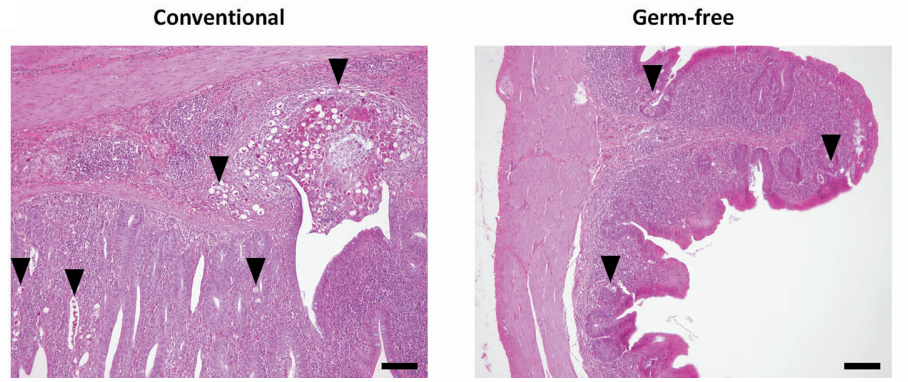

FIGURE 5 | The absence of gut microbiota has no effect on E. tenella sporozoite invasion capacity but alters its development. (A) The absence of microbiota had no effect on E. tenella sporozoite invasion capacity. Conventional and germ-free chickens were cloacally inoculated with $E$. tenella sporozoites expressing a nano luciferase reporter gene ( $10^{6}$ sporozoites per animal). Caecal tissues were washed in PBS and luciferase activity was detected $16 \mathrm{~h}$ post-infection. Medians are represented (Mann-Whitney; $n=10$; non-significant). No significant differences between germ-free and conventional chickens were observed. (B) The absence of microbiota alters the development of $E$. tenella. Conventional and germ-free chickens were cloacally inoculated with Et-INRAE sporozoites ( $\left.8 \times 10^{4}\right)$. On the left panel: Oocysts were counted in caecal contents at day 7 post-infection. On the right panel: the parasite load in caecal tissues at day 7 post-infection was determined by RT-qPCR. The expression of housekeeping parasite specific genes, Et18S and Etprofilin were expressed relative to Gallus gallus housekeeping genes g10 and gapdh using the $2^{-\Delta \mathrm{Ct}}$ formula: Ct Et housekeeping genes - Ct host housekeeping genes. Medians are represented (Mann-Whitney; $\mathrm{n} \geq 8$; ${ }^{\star \star} P<0.01$; ${ }^{* * * *} P<0.0001$ between germ-free and conventional chickens). (C) Representative histological images at day 7 post-infection showing lower parasite load in caecal tissues in germ-free chickens compared to conventional chickens. Histological analysis was performed on tissue sections stained with Hemalun Eosin Saffron; scale = $100 \mu \mathrm{m}$.

\section{DATA AVAILABILITY STATEMENT}

The raw data supporting the conclusions of this article will be made available by the authors, without undue reservation.

\section{ETHICS STATEMENT}

The animal study was reviewed and approved by CEEA Val de Loire $\mathrm{n}^{\circ} 19$; 2018-04-26 (APAFIS $\mathrm{N}^{\circ} 13904$ ).

\section{AUTHOR CONTRIBUTIONS}

FB designed the experiments. PG, GS, GF, ASa, AN, and FB performed the experiments. EG provided the conventional and germ-free chickens. CS and NL performed the bacteriological controls. AN provided the transgenic parasite Et-INV. CR, NL, TL performed the histology on tissues. FB, PG, GS analyzed the data. FB, PG, GS, SL-L, FL, CS discussed the data. FB, GS, ASi, SL-L, FL, RG, CS, wrote and/or reviewed the manuscript.
All authors contributed to the article and approved the submitted version.

\section{FUNDING}

This study was funded by the Région Centre Val de Loire, France (APR-IA “INTEGRITY” 2017-2019).

\section{ACKNOWLEDGEMENTS}

We are thankful to T. Chaumeil, M. Renouard, S. Lavillatte, P. Cousin, A. Faurie, O. Dubes, M. Girault from the Infectiology of Farm, Model and Wildlife Animals Facility (PFIE), for the animal care and skills with the animal experiments. The authors are grateful to the breeders and the society Boyé accouvage (La Boissière en Gâtine, France) for the supply of fertilized eggs. We also acknowledge Y. Le Vern for flow cytometry sorting of transgenic oocysts (INRAE, Université de Tours, UMR ISP, Platform IMI, F-37380, Nouzilly, France). We are grateful to P. Quéré (INRAE, Université de Tours, UMR ISP F-37380, Nouzilly, France) who kindly reviewed and gave suggestions for the manuscript. 


\section{REFERENCES}

Al-Sheikhly, F., and Al-Saieg, A. (1980). Role of coccidia in the occurrence of necrotic enteritis of chickens. Avian Dis. 24, 324-333. doi: 10.2307/1589700

Blake, D. P., Knox, J., Dehaeck, B., Huntington, B., Rathinam, T., Ravipati, V., et al. (2020). Re-calculating the cost of coccidiosis in chickens. Vet. Res. 51, 115. doi: $10.1186 / \mathrm{s} 13567-020-00837-2$

Bradley, R. E., and Radhakrishnan, C. V. (1973). Coccidiosis in chickens: obligate relationship between Eimeria tenella and certain species of cecal microflora in the pathogenesis of the disease. Avian Dis. 17, 461-476. doi: $10.2307 / 1589145$

Cheled-Shoval, S. L., Gamage, N. S., Amit-Romach, E., Forder, R., Marshal, J., Van Kessel, A., et al. (2014). Differences in intestinal mucin dynamics between germ-free and conventionally reared chickens after mannan-oligosaccharide supplementation. Poult. Sci. 93, 636-644. doi: 10.3382/ps.2013-03362

Cirimotich, C. M., Dong, Y., Clayton, A. M., Sandiford, S. L., Souza-Neto, J. A., Mulenga, M., et al. (2011a). Natural microbe-mediated refractoriness to Plasmodium infection in Anopheles gambiae. Science 332, 855-858. doi: 10.1126/science. 1201618

Cirimotich, C. M., Ramirez, J. L., and Dimopoulos, G. (2011b). Native microbiota shape insect vector competence for human pathogens. Cell Host Microbe 10, 307-310. doi: 10.1016/j.chom.2011.09.006

Crhanova, M., Hradecka, H., Faldynova, M., Matulova, M., Havlickova, H., Sisak, F., et al. (2011). Immune response of chicken gut to natural colonization by gut microflora and to Salmonella enterica serovar enteritidis infection. Infect. Immun. 79, 2755-2763. doi: 10.1128/IAI.01375-10

Ferguson, D. J., Belli, S. I., Smith, N. C., and Wallach, M. G. (2003). The development of the macrogamete and oocyst wall in Eimeria maxima: immuno-light and electron microscopy. Int. J. Parasitol. 33, 1329-1340. doi: 10.1016/S0020-7519(03)00185-1

Ganas, P., Liebhart, D., Glosmann, M., Hess, C., and Hess, M. (2012). Escherichia coli strongly supports the growth of Histomonas meleagridis, in a monoxenic culture, without influence on its pathogenicity. Int. J. Parasitol. 42, 893-901. doi: 10.1016/j.ijpara.2012.07.007

Guitton, E., Faurie, A., Lavillatte, S., Chaumeil, T., Gaboriaud, P., Bussiere, F., et al. (2020). Production of germ-free fast-growing broilers from a commercial line for microbiota studies. J. Vis. Exp. 18 (160), e61448. doi: 10.3791/61148

Hegde, S., Rolls, B. A., Turvey, A., and Coates, M. E. (1982). Influence of gut microflora on the lymphoid tissue of the chicken (Gallus domesticus) and japanese quail (Coturnix coturnix japonica). Comp. Biochem. Physiol. 72A, 205-209. doi: 10.1016/0300-9629(82)90034-2

Huang, G., Tang, X., Bi, F., Hao, Z., Han, Z., Suo, J., et al. (2018a). Eimeria tenella infection perturbs the chicken gut microbiota from the onset of oocyst shedding. Vet. Parasitol. 258, 30-37. doi: 10.1016/j.vetpar.2018.06.005

Huang, G., Zhang, S., Zhou, C., Tang, X., Li, C., Wang, C., et al. (2018b). Influence of Eimeria falciformis infection on gut microbiota and metabolic pathways in mice. Infect. Immun. 86 (5), e00073-18. doi: 10.1128/IAI.00073-18

Lafont, J. P., Yvore, P., Bree, A., and Peloille, M. (1975). Pathogenic effect of Eimeria tenella and Eimeria acervulina in axenic and monoxenic chickens. Ann. Rech. Vet. 6 (1), 35-42.

Laurent, F., Mancassola, R., Lacroix, S., Menezes, R., and Naciri, M. (2001). Analysis of chicken mucosal immune response to Eimeria tenella and Eimeria maxima infection by quantitative reverse transcription-PCR. Infect. Immun. 69, 2527-2534. doi: 10.1128/IAI.69.4.2527-2534.2001

Liu, Y., Tian, X., He, B., Hoang, T. K., Taylor, C. M., Blanchard, E., et al. (2019). Lactobacillus reuteri DSM 17938 feeding of healthy newborn mice regulates immune responses while modulating gut microbiota and boosting beneficial metabolites. Am. J. Physiol. Gastrointest. Liver Physiol. 317, G824-G838. doi: 10.1152/ajpgi.00107.2019
Long, S. L., Gahan, C. G. M., and Joyce, S. A. (2017). Interactions between gut bacteria and bile in health and disease. Mol. Aspects Med. 56, 54-65. doi: 10.1016/j.mam.2017.06.002

Macdonald, S. E., Nolan, M. J., Harman, K., Boulton, K., Hume, D. A., Tomley, F. M., et al. (2017). Effects of Eimeria tenella infection on chicken caecal microbiome diversity, exploring variation associated with severity of pathology. PloS One 12, e0184890. doi: 10.1371/journal.pone.0184890

Madsen, D., Beaver, M., Chang, L., Bruckner-Kardoss, E., and Wostmann, B. (1976). Analysis of bile acids in conventional and germ-free rats. J. Lipid Res. 17, 107-111. doi: 10.1016/S0022-2275(20)36993-5

Oakley, B. B., Lillehoj, H. S., Kogut, M. H., Kim, W. K., Maurer, J. J., Pedroso, A., et al. (2014). The chicken gastrointestinal microbiome. FEMS Microbiol. Lett. 360, 100-112. doi: 10.1111/1574-6968.12608

Qi, Z., Shi, S., Tu, J., and Li, S. (2019). Comparative metagenomic sequencing analysis of cecum microbiotal diversity and function in broilers and layers. 3 Biotech. 9, 316. doi: 10.1007/s13205-019-1834-1

Rolls, B. A., Turvey, A., and Coates, M. E. (1978). The influence of the gut microflora and of dietary fibre on epithelial cell migration in the chick intestine. Br. J. Nutr. 39, 91-98. doi: 10.1079/BJN19780015

Shirley, M. W. (1995). Eimeria species and strains of chicken. Biotechnology-Guidelines Techniques Coccidiosis Res. Luxembourg Eur. Commission DGXII, 1-24.

Stecher, B., Chaffron, S., Kappeli, R., Hapfelmeier, S., Freedrich, S., Weber, T. C., et al. (2010). Like will to like: abundances of closely related species can predict susceptibility to intestinal colonization by pathogenic and commensal bacteria. PloS Pathog. 6, e1000711. doi: 10.1371/journal.ppat.1000711

Swale, C., Bougdour, A., Gnahoui-David, A., Tottey, J., Georgeault, S., Laurent, F., et al. (2019). Metal-captured inhibition of pre-mRNA processing activity by CPSF3 controls Cryptosporidium infection. Sci. Transl. Med. 11 (517), eaax7161. doi: 10.1126/scitranslmed.aax7161

Visco, R. J., and Burns, W. C. (1972a). Eimeria tenella in bacteria-free and conventionalized chicks. J. Parasitol. 58, 323-331. doi: 10.2307/3278096

Visco, R. J., and Burns, W. C. (1972b). Eimeria tenella in bacteria-free chicks of relatively susceptible strains. J. Parasitol. 58, 586-588. doi: 10.2307/3278209

Walker, R. A., Sharman, P. A., Miller, C. M., Lippuner, C., Okoniewski, M., Eichenberger, R. M., et al. (2015). RNA Seq analysis of the Eimeria tenella gametocyte transcriptome reveals clues about the molecular basis for sexual reproduction and oocyst biogenesis. BMC Genomics 16, 94. doi: 10.1186/s12864-015-1298-6

Wei, S., Morrison, M., and Yu, Z. (2013). Bacterial census of poultry intestinal microbiome. Poult. Sci. 92, 671-683. doi: 10.3382/ps.2012-02822

Yan, W., Liu, X., Shi, T., Hao, L., Tomley, F. M., and Suo, X. (2009). Stable transfection of Eimeria tenella: constitutive expression of the YFP-YFP molecule throughout the life cycle. Int. J. Parasitol. 39, 109-117. doi: 10.1016/j.ijpara.2008.06.013

Yang, X., Brisbin, J., Yu, H., Wang, Q., Yin, F., Zhang, Y., et al. (2014). Selected lactic acid-producing bacterial isolates with the capacity to reduce Salmonella translocation and virulence gene expression in chickens. PloS One 9, e93022. doi: 10.1371/journal.pone.0093022

Conflict of Interest: The authors declare that the research was conducted in the absence of any commercial or financial relationships that could be construed as a potential conflict of interest.

Copyright (c) 2021 Gaboriaud, Sadrin, Guitton, Fort, Niepceron, Lallier, Rossignol, Larcher, Sausset, Guabiraba, Silvestre, Lacroix-Lamandé, Schouler, Laurent and Bussière. This is an open-access article distributed under the terms of the Creative Commons Attribution License (CC BY). The use, distribution or reproduction in other forums is permitted, provided the original author(s) and the copyright owner(s) are credited and that the original publication in this journal is cited, in accordance with accepted academic practice. No use, distribution or reproduction is permitted which does not comply with these terms. 